REVIEW

\title{
The radiology of chronic lung disease in children
}

\author{
U G Rossi, C M Owens
}

Arch Dis Child 2005;90:601-607. doi: 10.1136/adc.2004.051383

Chronic lung disease (CLD) in children represents a heterogeneous group of many distinct clinicopathological entities. The prevalence of CLD has increased in the past decade because of the more advanced and intensive respiratory support provided for compromised children and additionally the overall improved survival of preterm babies. The disorders which constitute CLD generally have a slow tempo of progression over many months or even years. The most common causes of CLD in children are cystic fibrosis (CF), and other causes of bronchiectasis (such as immunodeficiency, and in the third world, postinfective bronchiectasis, for example, measles), bronchopulmonary dysplasia (BPD) (or lung disease of prematurity), asthma, chronic gastro-oesophageal reflux/ aspiration pneumonitis, and constrictive obliterative bronchiolitis.

See end of article for authors' affiliations

....................

Correspondence to: Dr C M Owens, X-Ray Department, Great Ormond Street Children's Hospital, Great Ormond Street, London WC1N 3JH, UK; owensc@gosh. nhs.uk

Accepted 28 October 2004
$\mathrm{T}$ his article will address key radiological features of the disorders which constitute chronic lung disease in children and attempt diagnostic imaging modalities indicated in managing these children.

\section{IMAGING THE RESPIRATORY SYSTEM IN CHRONIC LUNG DISEASE IN CHILDREN}

To make sense of any clinical problem, integrating the clinical information is essential. Specific features to be delineated in the clinical context include the child's age, sex, current and previous systemic illnesses, immunodeficiency states, medications, family history, along with a good clinical examination.

Imaging has many roles: the first is to establish or substantiate the diagnosis and ascertain whether further (often more invasive) examinations are required; the second is to determine the extent of the disease, its natural history, and the effects of therapy.

\section{CHEST RADIOGRAPH}

The natural contrast between the air and the makes the chest radiograph the first line technique for most patients despite recent advances in imaging. Younger children are usually examined supine and older cooperative children can be examined erect. The routine use of lateral chest radiographs is unnecessary in paediatrics, but this projection should be reserved for certain clinical situations. An expiration film, in selected cases, can be taken to show good compliance of the lungs, suggesting that no to guide the rationale for use of the various respiratory airways, and the low radiation dose, over-inflation or air trapping is present. The radiologist's armamentarium includes the plain chest radiograph, ultrasound, fluoroscopy, computed tomography, magnetic resonance imaging, and nuclear medicine scintigraphy.

\section{LUNG CT (SPIRAL/VOLUMETRIC AND HIGH RESOLUTION)}

When the chest radiograph is non-specific, high resolution computed tomography (HRCT) has been shown in adults ${ }^{1-3}$ and in children ${ }^{4}$ to be more sensitive (it can detect abnormalities when the chest radiograph is normal); it also shows greater accuracy in characterising diseases. HRCT usually involves sampling $1 \mathrm{~mm}$ sections of the lung at 10-15 mm intervals, and examining on a high spatial resolution algorithm with a wide window width.

HRCT is the imaging modality of choice for the morphological assessment of pulmonary parenchyma with excellent spatial resolution. The trade-off in increased sensitivity and specificity of HRCT over chest radiography is related to radiation dose (which is higher); however, conventional spiral or volumetric computed tomography (CT) has an even higher radiation burden than HRCT. The use of low dose $(50 \mathrm{~mA}, 0.75 \mathrm{~s})$ limited ( $1 \mathrm{~mm}$ slices every $10-20 \mathrm{~mm}$ ) HRCT in inspiration with three expiratory supplementary scans allows accurate assessment of the presence and extent of diffuse lung disease at a dose equivalent to approximately $10-15$ chest radiographs. This compares to a dose for volumetric chest CT (which acquires a whole spiral volume of lung) of approximately 50 chest radiographs if low dose paediatric protocols are adhered to.

If a child is unable to breathhold, the scans can be performed during quiet breathing and decubitus scans replace expiratory scans (the dependent lung behaving as the "expiratory lung" and the non-dependent lung as the "inspiratory lung"). ${ }^{5}$

The clinical indications for HRCT in children are delineation of lung anatomy, study of both interstitial and airways disease, and dynamic studies to assess air trapping when expiratory/ decubitus images are used.

Advances in volumetric or spiral CT technology, particularly the development of multislice scanners, have resulted in faster scan acquisition times, and this, combined with reduction in the tube current, can allow the radiation dose to be kept to a minimum. However, radiation dose is

Abbreviations: BPD, bronchopulmonary dysplasia; $C F$, cystic fibrosis; CLD, chronic lung disease; CT, computed tomography; GOR, gastro-oesophageal reflux; HRCT, high resolution computed tomography; $M R I$, magnetic resonance imaging; US, ultrasound; VQ, ventilation perfusion 


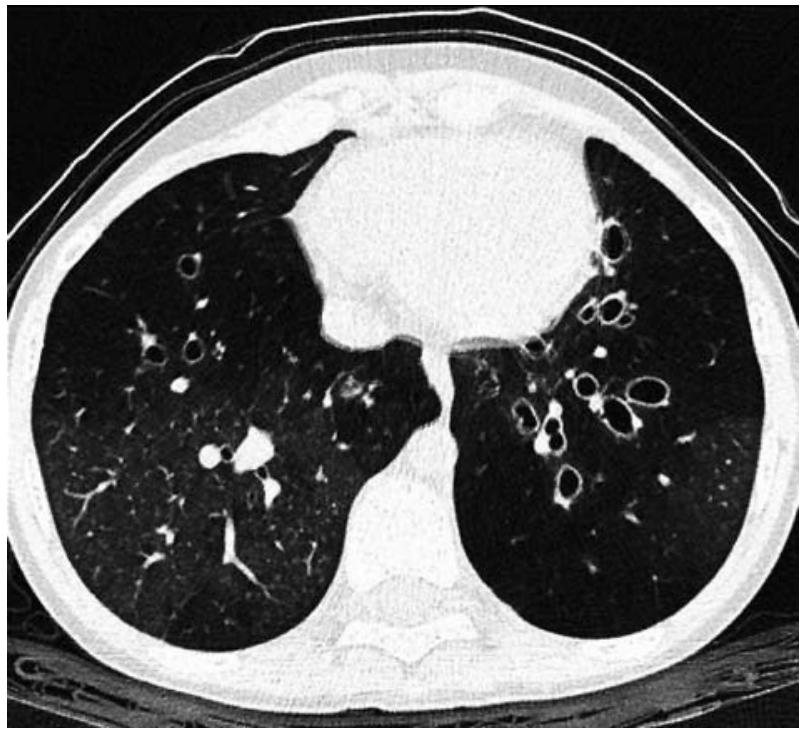

Figure 1 HRCT slice in a child with bronchiectasis shows bronchial dilatation with mild bronchial wall thickening (large airways disease), as well as almost globally hypoattenuating lung parenchyma and associated hypovascularity (small airways disease).

always significantly greater for CT than for a plain radiograph. It is vital that children are specifically catered for when undergoing chest CT as the doses for volumetric CT can be as much as 500 chest radiographs equivalent if an adult protocol is inadvertently used. This compares to approximately 50 chest radiographs if a low dose paediatric protocol is followed.

The clinical indications for volumetric or spiral CT include studies when definition of the entire volume of thoracic contents are required, such as definition of lung anatomy in congenital abnormalities of the tracheo-bronchial tree and vascular anatomy, assessment of pulmonary metastasis, mediastinal pathology (for example, mediastinal tumours, vascular rings, and post-traumatic assessment).

Depending on the age of the patient, the technique is performed in babies under 8 weeks of age after a feed or in older children under light sedation, usually without the need for general anaesthesia. HRCT is the gold standard for imaging lung parenchymal structure.

\section{NUCLEAR MEDICINE SCINTIGRAPHY}

Radionuclide imaging (nuclear medicine) provides reliable functional information regarding lung perfusion and ventilation. Nuclear medicine ventilation lung scans can be performed using radioactive gases, such as xenon-133, xenon-127, or krypton-81m, or by using a radioactive aerosol, such as technetium-99m. Perfusion scanning to assess pulmonary blood flow to the lung is usually carried out with technetium-99m macro aggregates of albumin. The principal use of pulmonary scintigraphy in children is for the assessment of regional lung function. The main indications for lung scintigraphy in children with chronic lung disease
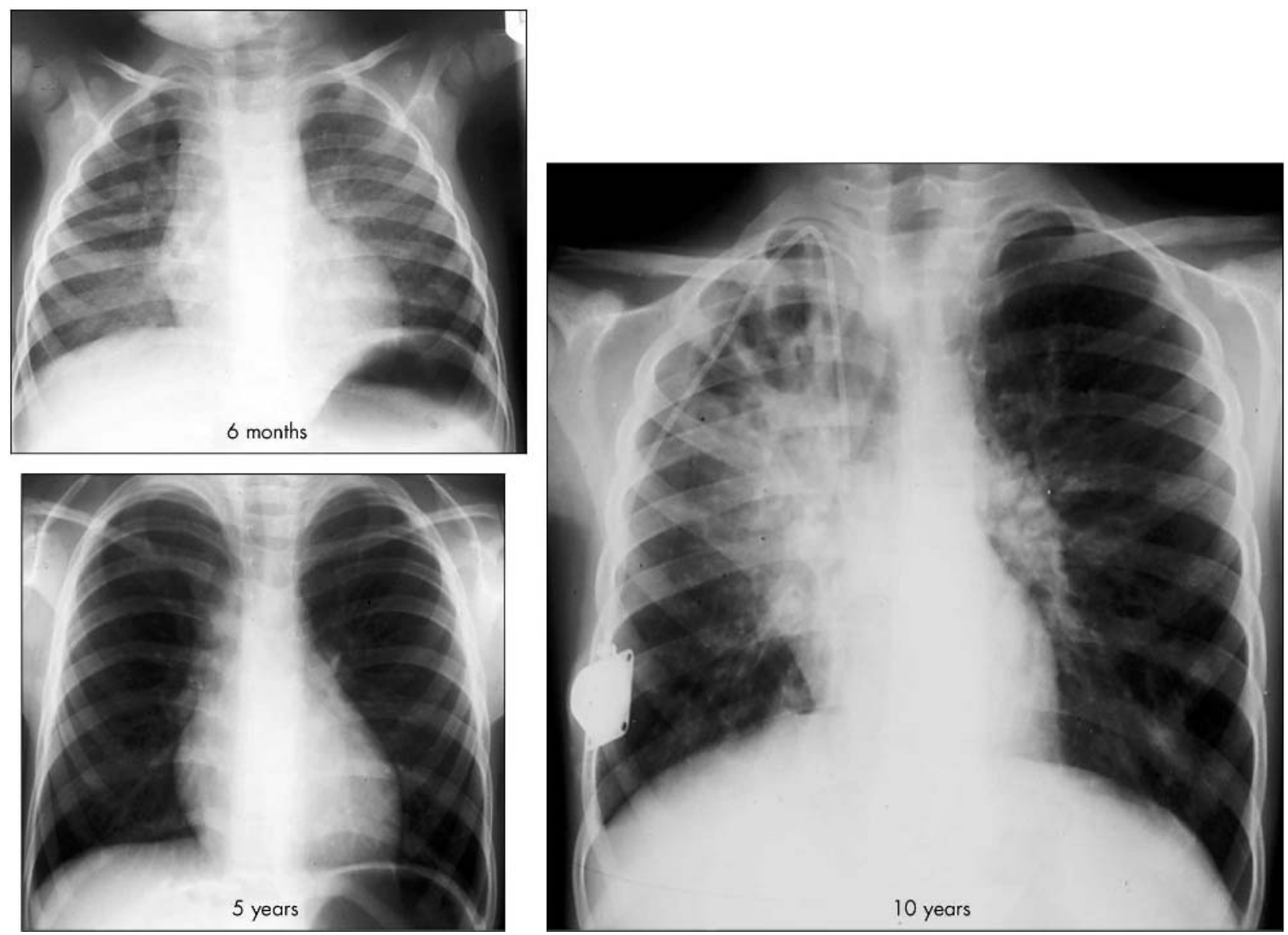

Figure 2 Chest radiographs in a child showing the serial changes of cystic fibrosis at 6 months, 5 years, and 10 years of age. There is progressive overinflation of the lungs with extensive bilateral bronchiectasis, particularly affecting the collapsed right upper lobe, where there is tubular dilatation of grossly abnormal bronchi. 


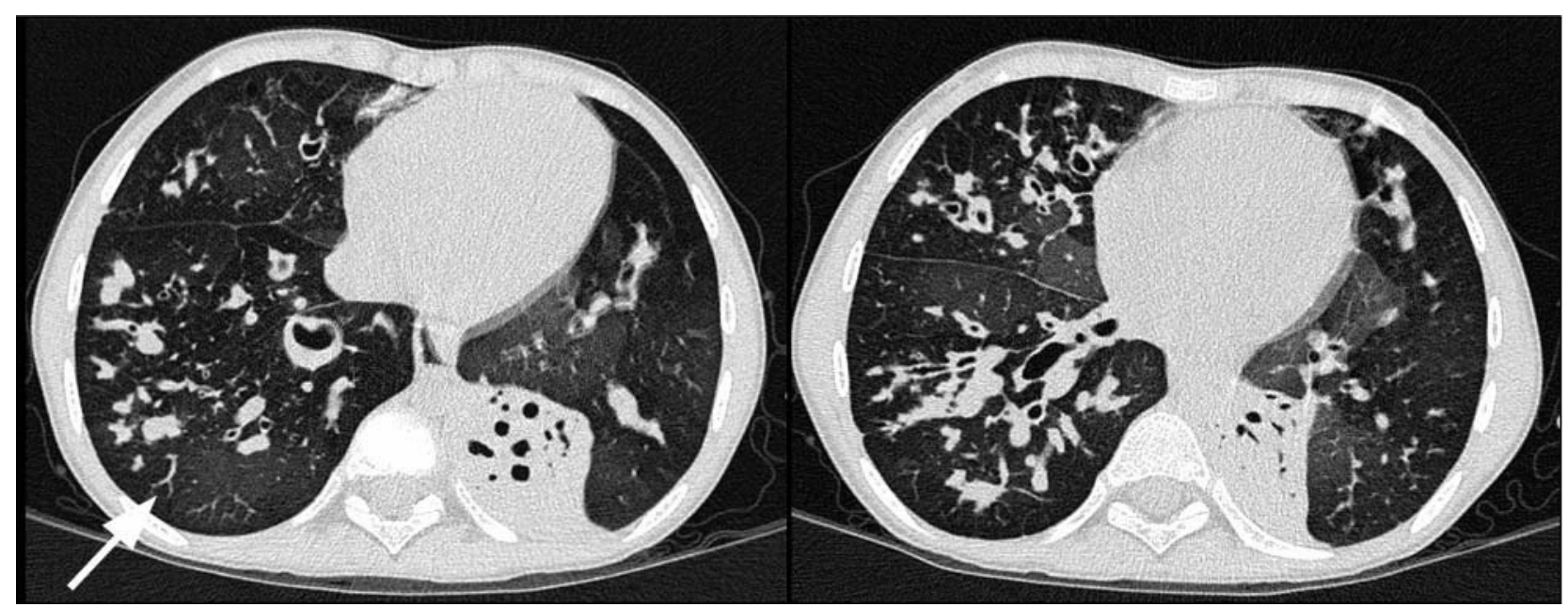

Figure 3 HRCT in a teenager with end stage cystic fibrosis shows mosaic attenuation, dilatation, and wall thickening of the bronchi, mucus within bronchi, and terminal branches with tree-in-bud sign (arrow). On expiration (right) there is accentuation of the mosaic pattern, confirming small airways obstruction.

are cystic fibrosis and bronchiectasis, as vascular diseases and pulmonary emboli are relatively rare compared to the adult population.

\section{MAGNETIC RESONANCE IMAGING}

The recent developments in magnetic resonance imaging (MRI) techniques to be utilised in the lung have allowed evaluation of lung function, but this is currently still under investigation for its clinical utility. Conventional MRI using the intravascular contrast agent (gadolinium) administered intravenously is helpful for visualisation of pulmonary vasculature and assessment of pulmonary perfusion. For qualitative visualisation as well as quantitative and functional data regarding ventilation, Gd-chelates, oxygen, or indeed other nuclei such as helium-3 or xenon-129 (inert gases) by aerosol can be used. MRI techniques are very expensive and take a long time to perform compared to nuclear medicine.

In conclusion, MRI is useful for assessment of vascular and mediastinal anatomy, and although a very attractive modality as there is no radiation burden, currently has a limited role outside of research centres in lung parenchymal imaging as the inherent poor signal to noise ratio renders the images of poor diagnostic quality.

\section{ULTRASONOGRAPHY}

Ultrasound (US) has a relatively limited role imaging the lung as the presence of air in the normal lung precludes transmission of the soundbeam through the normal lung parenchyma. However, US is the method of choice for differentiating the presence and consistency of pleural fluid such as an assessment of parapneumonic effusion or empyema. US can also potentially differentiate between a sub-pleural mass and lung collapse in a child with complete or partial opacification within the hemithorax seen on a chest radiograph. US is also the method of choice for differentiating solid from cystic pleural collections and assessing the viability of pleural drainage.

\section{INVASIVE CONTRAST BRONCHOGRAPHY}

Nowadays, invasive bronchography is relatively infrequently performed, as bronchiectasis and tracheobronchial anatomy are very easily studied using computed tomography. HRCT/ spiral CT has superseded bronchography in most cases and is less invasive with excellent resolution of airways anatomy, allowing the simultaneous evaluation of large and distal airways at the same sitting.

\section{IMAGING SUBTYPES OF CLD IN CHILDREN Bronchiectasis}

Bronchiectasis is defined as irreversible dilatation of the bronchial tree. This remains an important and relatively common cause of pulmonary disability. HRCT has revolutionised imaging of the bronchi and has superseded the chest radiograph and bronchogram in the diagnosis of bronchiectasis. It has enabled a better understanding of the association between the clinical features of the disease and the structural abnormalities in the airways, and has an important role to play in understanding the pathogenesis and natural history of bronchiectasis. In the early part of the 20th century, bronchiectasis was common, due to the high prevalence of childhood pulmonary infections, in particular pertussis, influenza, and tuberculosis. Vaccination against viral illness and the effect of treatment of bacterial pneumonia with antibiotics has meant that the classical form of bronchiectasis, typified by copious sputum associated with finger clubbing, is now relatively infrequently encountered in the UK. However, there is a resurgence of interest in the condition. There is much confusing literature regarding the increase sensitivity and specificity of HRCT over chest radiography in assessing bronchiectasis. In a study by Currie and colleagues, ${ }^{6}$ chest radiography was abnormal in only 9 of 19 patients with positive bronchography-that is, chest radiography sensitivity is poor at $47 \%$ of patients with proven bronchiectasis.

A study in 1987 by Silverman and Godwin ${ }^{7}$ showed that there was HRCT evidence of bronchiectasis in 14 patients who had normal chest radiographs-that is, CT was more sensitive in the diagnosis of bronchiectasis. There is no doubt that CT is useful for monitoring the response to therapy in patients with cystic fibrosis ${ }^{8}$ and those with bronchiectasis resulting from congenital immunodeficiency, particularly when they are treated with intravenous immunoglobulin. Currently HRCT is the gold standard in assessing bronchiectasis, particularly when assessing response to intervention such as antibiotics and intravenous immunoglobulin therapy.

\section{Cystic fibrosis}

Cystic fibrosis (CF) is an autosomal recessive multisystem disorder. CF is the most common cause of pulmonary insufficiency in the first three decades of life. ${ }^{90}$ The mechanisms by which this leads to lung disease are not 

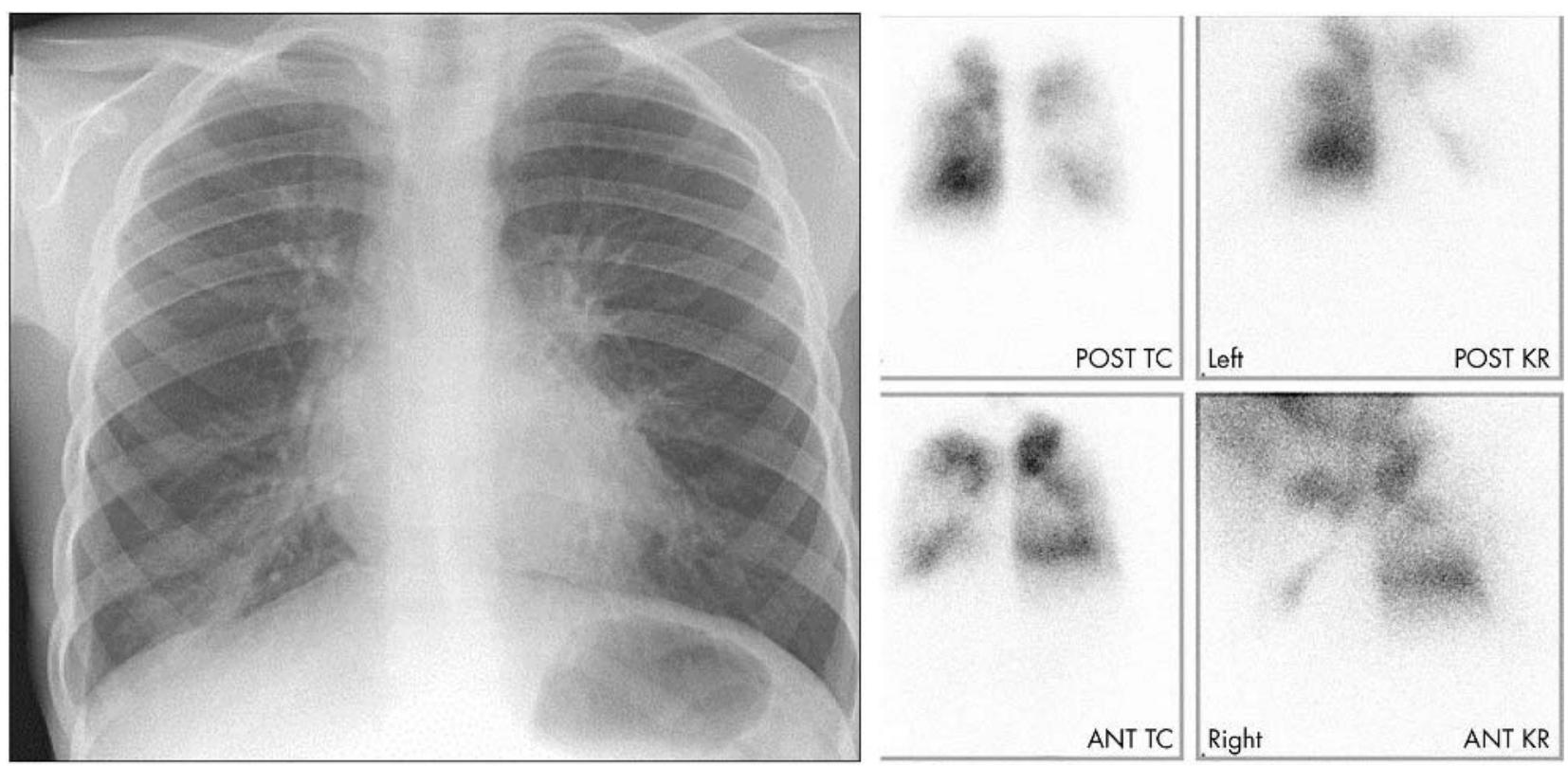

Figure 4 Chest radiograph showing mild bronchial thickening in a child with cystic fibrosis who had disproportionately severe respiratory functional impairment. VQ scans showed numerous matched ventilation and perfusion defects, explaining the child's clinical status.

entirely understood. The low water content of airway mucus is at least partially responsible, resulting in decreased mucous clearance, with resultant mucous plugging of the airways, and an increased incidence of bacterial airway infection. Bronchial wall inflammation progressing to secondary bronchiectasis is universal in patients with long standing $\mathrm{CF}$, and is commonly visible on the plain chest radiograph. ${ }^{11}$

Chest radiography findings in patients who have early or mild disease may be subtle, and bronchiectasis, especially in young patients, cannot be accurately defined using the chest radiograph, which is relatively insensitive. An early chest radiography finding is hyperinflation of the lungs, representing obstruction of the small airways, causing air trapping (fig 2). Thickening of the wall of the right upper lobe bronchus, best seen on the lateral radiograph, can also be an early sign of disease. ${ }^{12}$ Clinical findings (including a positive sweat test) and chest radiography are generally sufficient for the clinical management of the patient affected by CF.

High resolution computed tomography (HRCT) of the chest is the most useful technique in the identification of early lung abnormalities in patients with CF. In a study by Lynch and colleagues $^{13}$ of 12 asymptomatic paediatric patients with $\mathrm{CF}$, chest radiographs were normal in 7 of 12, whereas HRCT was normal in only 2 of 12 . The authors concluded that HRCT is significantly more sensitive in the definition of the affected airways. Bronchiectasis is the most frequent pathological finding on HRCT. In older children, adolescents, and young adults, bronchiectasis has been reported to be present in $78-100 \%$ of patients affected by CF. ${ }^{14}{ }^{15}$ Long and colleagues ${ }^{16}$ in a study of 34 infants with CF (age, $2.4 \pm 1.4$ years) compared with 20 normal infants ( $1.8 \pm 1.4$ years) confirmed that bronchiectasis begins very early in the course of CF and showed that the dilatation of the bronchial tree increased with increasing patient age. In early CF bronchiectasis is more frequently seen on HRCT in the upper lobes compared to the lower lobes.

Bronchial wall thickening and peribronchial interstitial thickening are also common in patients who have CF. The thickening is generally more evident than bronchial dilatation in patients who have early disease and may be seen independent of bronchiectasis. This occurs because the thickening of the airways reflects the inflammatory process that can be present in airways of all sizes.

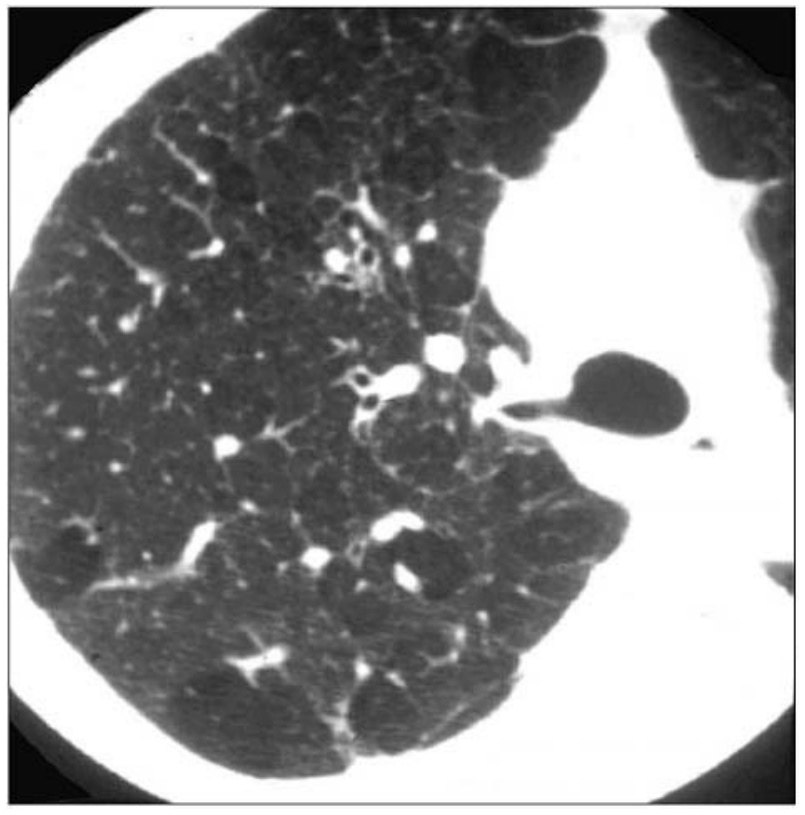

Figure $5 \mathrm{HRCT}$ in a child born prematurely who had been in intensive care over a long period. There are features of bronchopulmonary dysplasia: widespread mosaic attenuation (corresponding to areas of air trapping on subsequent expiratory scan) and architectural distortion.

Mucous plugging is also common and easily visualised with HRCT in all pulmonary lobes. In some patients, dilated small airways filled with mucus, pus, or peribronchiolar inflammation create nodular centrilobular opacities that are called "tree-in-bud" (fig 3).

Another early sign is lobular or segmental parenchymal inhomogeneity (mosaic perfusion which reflects small airways disease and air trapping). The presence of mosaic perfusion is related to regional constrictive bronchiolitis. Mosaic perfusion can be seen in inspiration but is potentiated on expiratory images, leading to reduced inter- and intraobserver observation when reporting HRCT. 


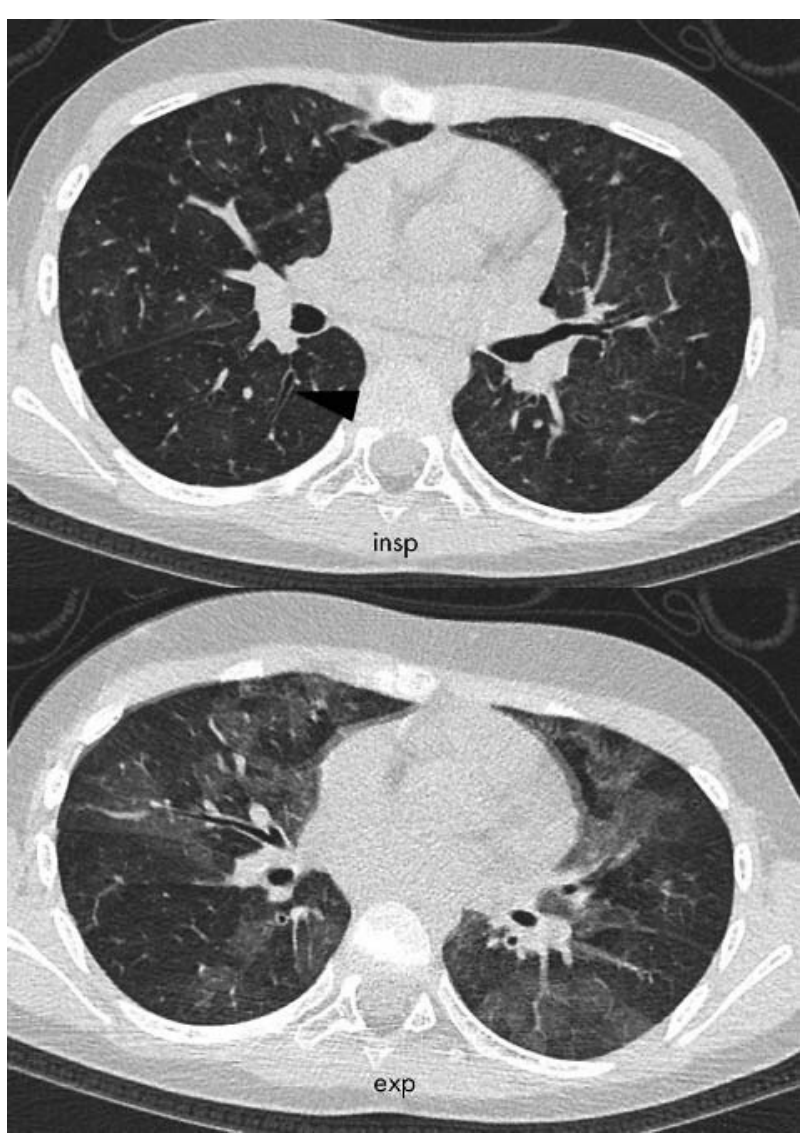

Figure 6 Inspiratory (upper) and expiratory (lower) scans in a child with constrictive obliterative bronchiolitis showing bronchiolectasis with mucus plugging at the centrilobular bronchi and mosaic attenuation due to reflex vasoconstriction which is more marked on the expiratory lower scans.

In summary, although an annual chest radiograph is currently advocated using the Conway and colleagues ${ }^{17}$ or Crispin-Norman scores, ${ }^{18}$ some centres advocate annual HRCT because of the increased sensitivity and specificity but with a questionable clinical management impact. The added benefit of HRCT in assessing small airways disease in CF has a good correlation with a functional impairment and is therefore useful. ${ }^{19}$ HRCT is also valuable in pre-transplant assessment of the lungs and for assessing the pleural status. Nuclear medicine scintigraphy can also provide useful information when the chest radiograph is relatively unremarkable in a child who has disproportionately severe clinical symptoms. VQ scans may show marked functional impairment in these patients with abnormal matched ventilation and perfusion defects (fig 4).

\section{BRONCHOPULMONARY DYSPLASIA}

Bronchopulmonary dysplasia (BPD) or lung disease of prematurity occurs in babies who have been subjected to mechanical ventilation as neonates. BPD was first described by Northway et al in $1967^{20}$ in babies who had been ventilated at high pressure and with high inspired oxygen concentrations. In more recent years, BPD is seen in preterm babies who have undergone more technically advanced ventilation at lower mean airway pressures and oxygen concentrations, but who undergo prolonged ventilation often because of severe prematurity.

Due to technical advances in antenatal and fetal therapy, there has been an increase in survival of extremely premature neonates, and yet despite new ventilation techniques, the incidence of BPD continues to increase.

Whereas in most children with mild to moderate BPD, radiographic findings and abnormalities of pulmonary function may be subtle and improve with age, ${ }^{21}$ less fortunate children suffer long term pulmonary complications including poor respiratory function, reduced exercise tolerance, and reactive airways disease.

The plain chest radiographic abnormalities reported in patients with BPD include hyper-expansion of the lung, multiple linear interstitial opacities, lung cysts, and emphysema. ${ }^{22}{ }^{23}$ However, chest radiographs often show only minor abnormalities in children affected by BPD despite significant ventilatory dysfunction.

HRCT (being more sensitive than the chest radiograph) shows areas of hyper-transradiancy, which consist of areas of low attenuation due to a reduction per unit area of pulmonary vasculature. Areas of hyperinflation tend to be multi-focal and often asymmetrical throughout the lungs. Linear opacities radiating from the periphery toward the hilum are present. ${ }^{24}$ Other imaging findings are bronchial wall thickening, decreased bronchus-to-pulmonary artery diameter ratios, ${ }^{24}$ and triangular sub pleural opacities, defined as small triangles with a pleural base and an internal apex. ${ }^{24}$ In most cases, linear opacities occur adjacent to the apex of subpleural triangular opacity (fig 4). The typical histological changes observed in BPD include: marked squamous metaplasia of large and small airways, peribronchial and peribronchiolar fibrosis, obliterating fibroproliferative bronchiolitis, and prominent hypertrophy of peribronchiolar smooth muscle. ${ }^{26}$ Aquino and colleagues, ${ }^{27}$ in a study on older children and adults with BPD, showed that in the expiratory HRCT scans $92 \%$ of the patients had evidence of air trapping. Large airway changes are also seen and the incidence of tracheo-bronchiomalacia and iatrogenic stenosis of the main airway is increased in BPD; both spiral CT and HRCT can be used to diagnose collapsed or segmental airway narrowing, although lesions may be missed or incorrectly diagnosed by either method. ${ }^{28}$ The diagnostic accuracy of these techniques is enhanced when a combination of both is used. Volumetric computer assisted reconstruction of airways and endoscopic images has enabled investigators to create three dimensional models of airways and airway abnormalities. ${ }^{29}$ This technique enables information to be gathered about all tracheobronchial and parenchymal anatomical abnormalities.

\section{ASTHMA}

Bronchial asthma is a heterogeneous disease characterised by reversible airway obstruction. This disease represents a chronic inflammatory process of the airways followed by healing, which may result in altered airway structure referred to as airway remodelling. ${ }^{30}$ This consists of a combination of bronchial wall thickening, caused by inflammation and mucosal oedema, connective tissue deposition in the extracellular matrix, and an increase of muscle mass and mucous gland numbers.

Radiographic findings in asthmatic patients include overinflation of the lungs, mild bronchial wall thickening manifest as peribronchial thickening; appearing as ring shadows when seen en face, or parallel tram lining when the bronchi are perpendicular to the radiographic beam. Areas of increased lung lucency and mild hilar prominence associated with the enlargement of the central pulmonary vessels due to transient pulmonary hypertension are also observed. Paganin and colleagues ${ }^{31}$ found that the chest radiograph showed the expected abnormalities of asthma in $37.8 \%$ of the 57 adult patients with chronic asthma. Zieverink and colleagues, ${ }^{32}$ in a retrospective study of 997 radiographs, 


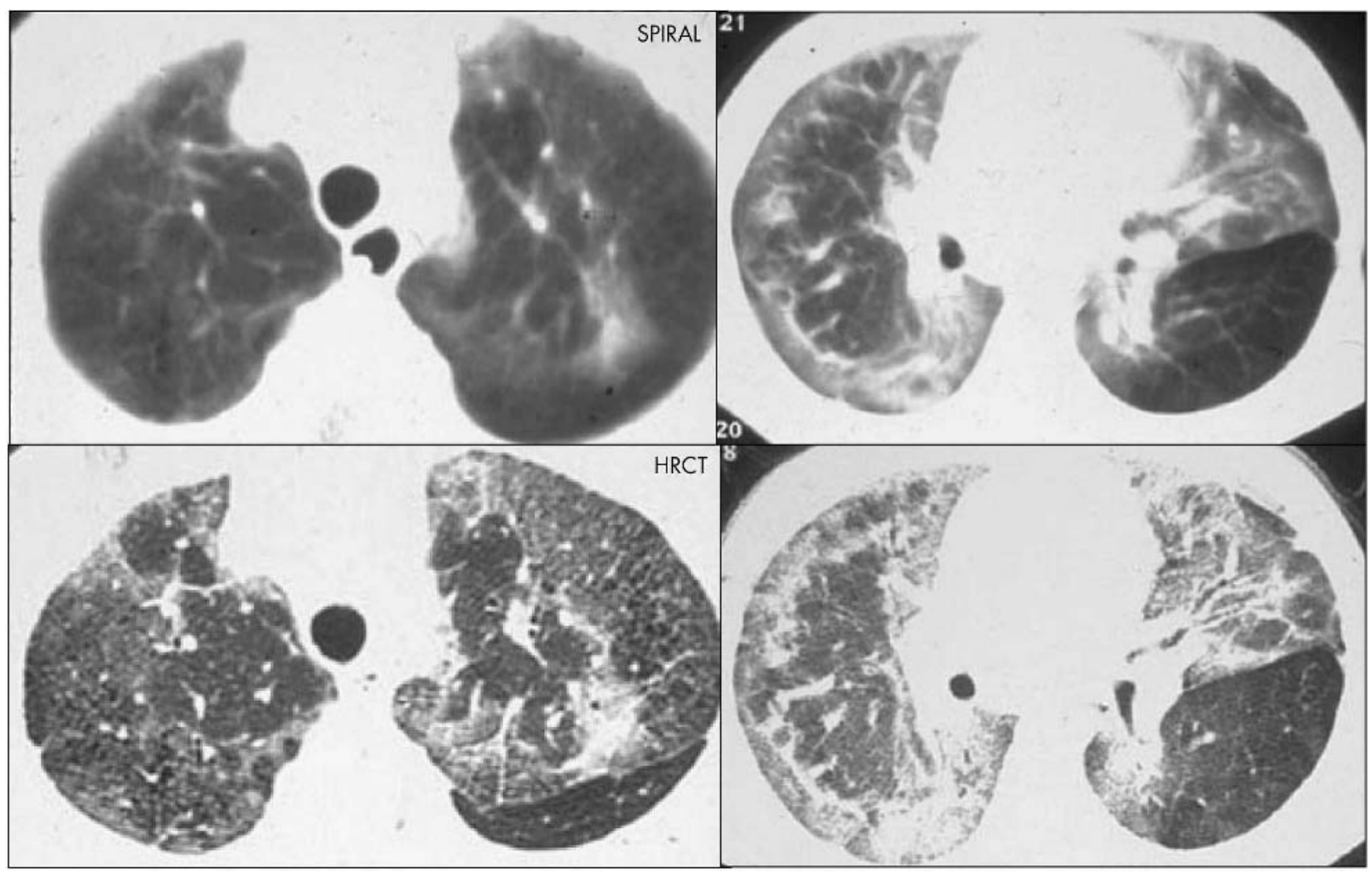

Figure 7 Spiral and HRCT in a child with unexplained respiratory distress. There are diffuse airway and interstitial changes with peripheral pulmonary fibrosis, best appreciated on HRCT (lower images) over spiral CT (upper images). A barium swallow showed gross gastro-oesophageal reflux and aspiration into the lungs. Lung biopsy confirmed the presence of numerous fat laden macrophages consistent with the diagnosis of aspiration pneumonitis and subsequent bronchiectasis pulmonary fibrosis.

showed that chest radiographs of asthmatic adults patients in an emergency room were abnormal in $2.2 \%$. On the contrary chest radiographs showed abnormal features in 13\% of asthmatic paediatric patients. Nevertheless, chest radiography or HRCT are rarely indicated in routine assessment of asthmatic patients, but can be used in association with clinical and lung function data to assess the severity of asthma in patients with persistent symptoms or when complications are suspected.

Goldin and colleagues, ${ }^{33}$ in an experimental study in asthmatic subjects and healthy controls, have measured bronchial diameter using HRCT after methacholine challenge, and then scanned following albuterol in patients with asthma and healthy control subjects. The studies showed that after methacholine there was a significant reduction in the diameter of the small bronchi in patients with asthma only.

HRCT findings are divided into reversible and irreversible abnormalities.

Reversible abnormalities include mucoid impactions, acinar opacification, and lobar collapse.

Mucoid impaction has been reported in as many as $21 \%$ of cases, ${ }^{31}$ but this abnormality may disappear after treatment.

Irreversible abnormalities include bronchial wall thickening and focal or diffuse hyperlucent areas.

Bronchial wall thickening is due to airway inflammation or airway remodelling. In children bronchial wall thickening visible on HRCT may reflect reversible or irreversible inflammation. Marchac and colleagues ${ }^{34}$ showed that inflammation persists in children with persistent symptoms with asthma which is difficult to treat, even in those patients with moderate symptoms, and these individuals may therefore have a higher risk of airway remodelling and complications. Awadh and colleagues ${ }^{35}$ showed that patients with asthma of different degrees of severity have more airway wall thickening than normal individuals. Furthermore, patients with more severe asthma have more bronchial wall thickening than those with mild disease.

In summary, radiology has a very limited role in the management of asthma and the chest radiograph is useful only when there is clinical evidence of an acute exacerbation to exclude infection or complications such as pneumothorax. At the present time, there is no role for HRCT in the routine management of asthmatic patients.

\section{CONSTRICTIVE (OBLITERATIVE) BRONCHIOLITIS}

Constrictive (obliterative) bronchiolitis is a severe indolent condition which presents clinically with a productive cough, dyspnoea, and deranged pulmonary function with an obstructive defect, a normal gas transfer (KCO), and air trapping shown as an increase in residual volume. The aetiological factors, which can result in this condition, include postrespiratory infections, particularly with adenovirus and mycoplasma pneumonia. Other predisposing conditions include connective tissue disorders and their therapy, and following bone marrow and heart lung transplantation.

Radiological features include often an unremarkable chest radiograph which belies abnormal HRCT; the hallmark features are patchy areas of hypertransradiant lung due to air trapping as a result of partial or complete airway obstruction by fibrous granulation tissue.

HRCT features include areas of hypertransradiancy from complete/partial airway obstruction by fibrous granulation tissue with reflex vasoconstriction and ground glass change due to vascular reperfusion of the more normal areas of lung. There is also evidence of bronchial wall thickening and dilatation (bronchiectasis). ${ }^{36}$ Decubitus (dependent lung 
acting as effective inspiratory phase of respiration) or expiratory scans increase the inter- and intra-observer agreement for assessing areas of hypertransradiancy due to air trapping ${ }^{37} 38$ (fig 6).

\section{CHRONIC GASTRO-OESOPHAGEAL REFLUX}

One of the most difficult areas of paediatric respiratory medicine is the relation between gastro-oesophageal reflux (GOR) and recurrent wheezing in infants and young children. Both occur independently, but when they occur in the same child, a relation between the two is often supposed. The major mechanism underlying GOR is transient lower oesophageal sphincter relaxation. Ing ${ }^{39}$ concluded that in adults GOR is associated with two of the heterogeneous groups of interstitial lung disease. The first, systemic sclerosis, is responsible for both pulmonary and oesophageal manifestations, and it remains difficult to determine the role that each has in the pathogenesis of the other. The second is idiopathic pulmonary fibrosis, which has a very high prevalence of GOR compared with both normal individuals and with other patients with interstitial lung disease without reflux.

HRCT features in children with systemic sclerosis include ground-glass opacification, subpleural micro-nodules, linear opacities, and honeycomb lung. ${ }^{5}$ In those children with untreated GOR unfortunate enough to progress to pulmonary fibrosis, this is characterised on HRCT by honeycombing, traction bronchiectasis, and ground glass opacity (fig 7).

GOR is also a potential trigger of asthma or reactive airways disease. The prevalence and severity of GOR in people with asthma are higher than in normal population. Mechanisms by which oesophageal acids induce bronchoconstriction in airway disease include a vagally mediated reflex, enhanced bronchial reactivity, and micro-aspiration. ${ }^{40}$

\section{SUMMARY}

It is important for the clinician and radiologist to work as a team in the radiological management of the expanding numbers of children with chronic lung disease, so that we continue to manage and match the most appropriate radiological procedure with each individual in order to ascertain the correct clinical diagnosis and management pathways in these patients.

The first line of investigation is the plain chest radiograph; when this is normal or inconclusive a low dose CT of the lungs acquired in either a volumetric or more often high resolution (non-contiguous) mode should be performed for a more sensitive assessment of the lung parenchymal detail. Nuclear medicine scintigraphy remains valuable when structural information requires supplementation with functional data regarding absolute or differential lung function.

MRI is a very attractive modality as there is no radiation burden, but currently has a limited role in lung parenchymal imaging as the inherent poor signal to noise ratio renders the images of poor diagnostic quality. MRI is useful in assessing cardiac and thoracic vascular anatomy and to assess the anatomy of the mediastinal structures.

\section{Authors' affiliations}

U G Rossi, C M Owens, Great Ormond Street Children's Hospital, London, UK

Competing interests: none declared

\section{REFERENCES}

1 Mathieson J, Mayo JR, Staples CA, et al. Chronic diffuse infiltrative lung disease: comparison of diagnostic accuracy of CT and chest radiography. Radiology 1989;171:111-16.

2 Grenier P, Valeyre D, Cluzel P, et al. Chronic diffuse interstitial lung disease: diagnostic value of chest radiography and high-resolution CT. Radiology $1991 ; 179: 123-32$.
3 Padley SP, Hansell DM, Flower CDR, et al. Comparative accuracy of highresolution computer tomography and chest radiography of diffuse lung disease. Clin Radiol 1991;44:222-4.

4 Lynch DA, Brasch RC, Hardy KA, et al. Paediatric pulmonary disease: assessment with high-resolution ultrafast CT. Radiology 1990;176:243-8.

5 Owens C. Radiology of diffuse interstitial pulmonary disease in children. Eur Radiol 2004; 14(4):L2-12.

6 Currie DC, Cooke JC, Morgan AD, et al. Interpretation of bronchograms and chest radiographs in patients with chronic sputum production. Thorax 1987;42:278-84.

7 Silverman PM, Godwin JD. CT/bronchographic correlations in bronchiectasis. J Comput Assist Tomogr 1987;1 1:52-6.

8 Brody AS. Scoring systems for CT in cystic fibrosis: who cares. Radiology 2004;231:296-8.

9 Wood BP. Cystic fibrosis. Radiology 1997;204:1-10.

10 Stern RC. The diagnosis of cystic fibrosis. N Engl J Med 1997;336:487-91.

11 Friedman PJ. Chest radiographic findings in the adults with cystic fibrosis. Semin Roentgenol 1987;22:114-24.

12 Reining JW, Sanchez FW, Thomason DM, et al. The distinctly visible right upper lobe bronchus on the lateral chest: a clue to adolescent cystic fibrosis. Pediatr Radiol 1985; 15:222-4

13 Lynch DA, Brasch RC, Hardy KA, et al. Pediatric pulmonary disease: assessment with high-resolution ultrafast CT. Radiology 1990;176:243-8.

14 Helbich TH, Heinz-Peer G, Eichler I, et al. Cystic fibrosis: CT assessment of lung involvement in children and adults. Radiology 1999;213:537-44.

15 Marchant JM, Masel JP, Dickenson FL, et al. Application of chest highresolution computer tomography in young children with cystic fibrosis. Pediatr Pulmonol 2001;31:24-9.

16 Long FR, Williams RS, Castile RG. Structural airway abnormalities in infants and young children with cystic fibrosis. J Pediatr 2004; 144:154-61.

17 Conway SP, Pond MN, Bowler I, et al. The chest radiograph in cystic fibrosis: a new scoring system compared with the Crispin-Norman and Brasfield scores. Thorax 1994;49:860-2.

18 Chrispin AR, Norman AP. The systematic evaluation of the chest radiograph with cystic fibrosis. Pediatr Radiol 1974;2:101-5.

19 Pim A de Jong, Ottink MD. Pulmonary disease assessment in cystic fibrosis: comparison of CT scoring systems and value of bronchial and arterial dimension measurements. Radiology 2004;231:434-9.

20 Northway WH Jr, Rosan RC, Porter DY. Pulmonary disease following respiratory therapy of hyaline membrane disease. N Engl J Med 1967;276:357-68.

21 Hakulinen AL, Jarvenpaa AL, Turpeinen $M$, et al. Diffusing capacity of the lung in school-aged children born very preterm, with and without bronchopulmonary dysplasia. Pediatr Pulmonol 1996;21:353-60.

22 Northway WH Jr, Moss RB, Carlisle KB, et al. Late pulmonary sequelae of bronchopulmonary dysplasia. N Engl J Med 1990;323:1793-9.

23 Griscom NT, Wheeler WB, Sweezey NB, et al. Bronchopulmonary dysplasia: radiographic appearance in middle childhood. Radiology 1989;171:811-14.

24 Oppenheim C, Mamou-Mani T, Sayegh N, et al. Bronchopulmonary dysplasia: value of $\mathrm{CT}$ in identifying pulmonary sequelae. Am J Roentgenol 1994; 163:169-72.

25 Howling SW, Northway WH Jr, Hansell DM, et al. Pulmonary sequelae of bronchopulmonary dysplasia survivors: high-resolution CT findings. Am J Roentgenol 2000;174:1323-6.

26 Stocker JT. Pathologic features of longstanding "healed" bronchopulmonary dysplasia. Hum Pathol 1986;17:943-61.

27 Aquino SL, Schechter MS, Chiles C, et al. High-resolution inspiratory and expiratory CT in older children and adults with bronchopulmonary dysplasia. Am J Roentgenol 1999;173:963-7.

28 Brody AS, Kuhn JP, Seidel FG, et al. Airway evaluation in children with use of ultrafast CT: piffalls and recommendations. Radiology 1991;178:181-4.

29 Dunham ME, Wolf RN. Visualizing the pediatric airway: three-dimensional modelling of endoscopic images. Ann Otol Rhinol Laryngol 1996;105:12-17.

30 Rennard SI. Repair mechanisms in asthma. J Allergy Clin Immunol 1996;98:S278-86.

31 Paganin F, Trussard V, Seneterre E, et al. Chest radiography and high resolution computed tomography of the lungs in asthma. Am Rev Respir Dis 1992;146:1084-7.

32 Zieverink SE, Harper AP, Holden RW, et al. Emergency room radiography of asthma: an efficacy study. Radiology 1982;145:27-9.

33 Goldin JG, McNitt-Gray MF, Sorenson SM, et al. Airway hyper reactivity: assessment with helical thin-section CT. Radiology 1998;208:321-9.

34 Marchac V, Emond S, Manou-Mani T, et al. Thoracic CT in pediatric patients with difficult-to-treat asthma. Am J Roentgenol 2002;179:1245-52.

35 Awadh N, Muller NL, Park CS, et al. Airway wall thickness in patients with near fatal asthma and control groups: assessment with high resolution computed tomographic scanning. Thorax 1998;53:248-53.

36 Hansell DM, Rubens MB, Padley SP, et al. Obliterative bronchiolitis: individual $\mathrm{CT}$ signs of small airways disease and functional correlation. Radiology 1997;203:721-6.

37 Worthy SA, Park CS, Kim JS, et al. Bronchiolitis obliterans after lung transplantation: high-resolution CT findings in 15 patients. Am J Roentgenol 1997; 169:673-7.

38 Bankier AA, Muylem AV, Knoop C, et al. Bronchiolitis obliterans syndrome in heart-lung transplant recipients: diagnosis with expiratory CT. Radiology $2001 ; 218: 533-9$

39 Ing AJ. Interstitial lung disease and gastroesophageal reflux. Am J Med $2001 ; 111: 41$ S-44S

40 Harding SM. Gastroesophageal reflux, asthma, and mechanisms of interaction. Am J Med 2001;111:8S-12S. 